\title{
Constructing the Overlay Network by Tuning Link Weights
}

\author{
Huijuan Wang and Piet Van Mieghem \\ Delft University of Technology, P.O. Box 5031, 2600 GA Delft, The Netherlands \\ Email: \{H.Wang, P.VanMieghem\}@ewi.tudelft.nl.
}

\begin{abstract}
When transport in networks follows the shortest paths, the union of all shortest path trees $G_{\cup s p t}$ can be regarded as the "transport overlay network". Overlay networks such as peer-to-peer networks or virtual private networks can be considered as a subgraph of $G \cup s p t$. We construct two types of $G_{\cup s p t}$ : (a) $G_{\cup s p t(\alpha)}$ where $\alpha$ is the extreme value index of polynomial link weights and (b) $G_{\cup \operatorname{spt}(\rho)}$ where $\rho$ is the correlation coefficient of the 2-dimensional correlated uniformly distributed link weights in QoS routing.

By tuning the extreme value index $\alpha$ of polynomial link weights, a phase transition occurs around a critical extreme value index $\alpha_{c}$ of the link weight distribution. If $\alpha>\alpha_{c}$, transport in the network traverses many links whereas for $\alpha<\alpha_{c}$, all transport flows over a critical backbone: the Minimum Spanning Tree (MST). In QoS routing with 2-dimensional link weights, as we decrease the correlation coefficient $\rho$ from 1 to -1 , the overlay $G \cup s p t$ becomes denser, and is equal to the substrate when $\rho=-1$. With the Erdös-Rényi random graph as the underlying topology, we show that the overlay $G_{\cup s p t(\rho)}$ is also close to an Erdös-Rényi random graph $G_{p}(N)$, an observation with potential for mobile and wireless ad-hoc networks. The existence of such a controllable transition in the overlay structure may allow network operators to steer and balance flows in their network.
\end{abstract}

\section{INTRODUCTION}

Routing in communication networks is based on shortest paths(or the best approximation due to e.g. the distracting influence of BGP) between any two nodes of the network. The resources of a network are most efficiently used when traffic follows shortest path [16]. Even for the Internet, it is a reasonable assumption, since roughly $80 \%$ of the routes seems to correspond to shortest paths.

In this paper, we study the overlay $G_{\cup s p t}$ formed by the union of all shortest path trees $S P T$ in a graph $G(N, L)$ with $N$ nodes and $L$ links, where a $S P T$ is the union of the shortest paths from one node to all the other nodes. The relation between the overlay $G_{\cup s p t}$ and the underlying graph or substrate $G(N, L)$ is shown in Figure 1. The overlay $G_{\cup s p t}$ can be regarded as the "transport overlay network" on top of the network topology or substrate. In the Internet, for example, traffic is carried along the overlay $G_{\cup s p t}$, composed of a fraction of the links in the underlying network, which is just the maximal part of the Internet that we can actually observe by traceroute measurements.

The importance of overlay networks is believed to grow in the future. One example of an overlay network is peerto-peer networks [21] with $n$ distributed systems sharing resources such as content, CPU cycles and storage, where $n$ is smaller than the number of nodes $N$ in the underlying

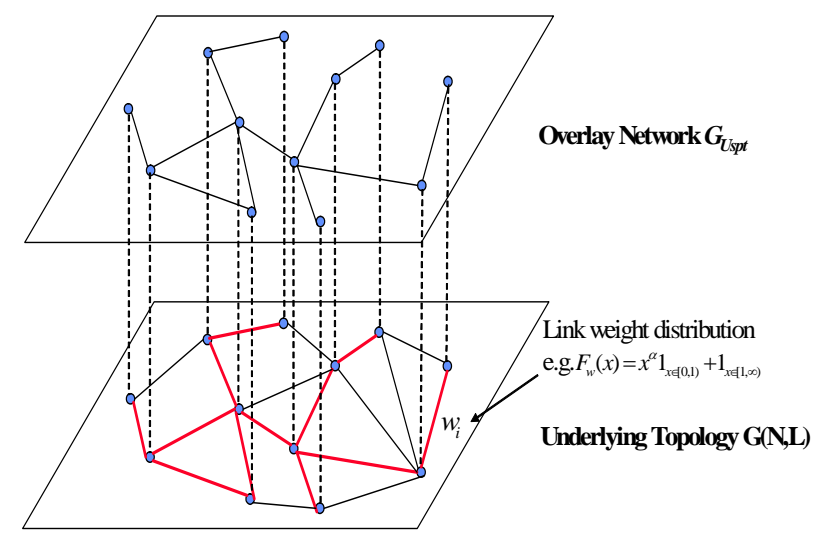

Fig. 1. The relation between the overlay network and the underlying topology.

network. The peer-to-peer overlay network can be regarded as a union of paths connecting these $n$ nodes. Another type of overlay network is a virtual private network (VPN), a private network that uses a public network (usually the Internet or the telephony network) to connect remote sites or users together. The physical networks traversed by both the peer-topeer and the VPN overlay networks are a subgraph of $G_{\cup s p t}$. The robustness in such overlay networks, the persistence of epidemics [2] and the vulnerability to node failures and attacks [10] are depending on structural properties of $G_{\cup s p t}$ that are studied in this paper.

The overlay $G_{\cup s p t}$, not the substrate, determines the network's performance: any link removed in $G_{\cup s p t}$ will definitely impact at least those flows of traffic that pass over that link. Here we show that, instead of changing the infrastructure of a network [22], the overlay network $G_{\cup s p t}$ can be controlled by varying the link weight structure. Current best-effort routing simply computes appropriate paths based on a single, relatively static measure (e.g. the delay, the monetary cost, etc.). Several quality-of-service (QoS) based networking frameworks (e.g., IntServ, DiffServ, MPLS) have been extensively investigated. QoS routing takes into account multiple measures including both the applications requirements' and the availability of network resources. We present two ways of constructing the overlay $G_{\cup s p t}$ : (a) one by changing the single link weight per link as in best-effort routing and (b) another by changing the link weight vector assigned to each link as in QoS routing [13].

The paper is outlined as follows. First, in Section II, 
we explain the modeling of the underlying topology, two schemes of tuning link weight structure and the algorithms for calculating the overlay $G_{\cup s p t}$. The structure of the $G_{\cup s p t}$ with uniformly distributed link weights is analyzed in Section III. The effect of these two link weight tuning schemes on the structure of the constructed overlay $G_{\cup s p t}$ are presented in Section IV and Section V. Finally, our results are summarized in Section VI.

\section{Modeling OF TOPOLOGY, LINK WEIGHT TUNING AND ROUTING ALGORITHMS}

In this paper, we consider the class of dense underlying topologies: the Erdös-Rényi random graph $G_{p}(N)$. Traditionally, complex networks have been modeled as Erdös-Rényi random graphs. Beside that, the Erdös-Rényi random graphs are reasonably accurate models for peer-to-peer networks [12] and ad-hoc networks [18]. The classes of lattice and power-law graphs will not be discussed and will only be mentioned when we demonstrate the uniform phase transition of the overlay network in Section IV. The main reason to omit these sparse graphs as substrates is that the overlay network is very close to the underlying topology. Besides, the so claimed power-law graph such as the Internet, the WWW, protein interaction and metabolic networks contains, in fact, more redundent links due to the limitation of its detection method [1], [3], [5], which is consistent with our dense underlying topology assumption.

We confine ourselves to additive and strict positive link weight measures (e.g. the delay, the monetary cost, etc.) such that the path weight equals the sum of the weights of links that constitute that path. Multiplicative measures (e.g. packet loss) can be transformed into additive weights by using the logarithm. In large networks, the weights or weight vectors of links are hardly correlated and can be considered as independent to a good approximation. Two ways of link weight tuning are investigated and two corresponding algorithms to find the shortest path or the optimal path are used:

(a) Each link is specified by a single weight measure. The overlay $G_{\cup s p t}$ is found by calculating the shortest paths between all node pairs with the Dijkstra algorithm [6]. Since the shortest path (SP) is mainly sensitive to the smaller, nonnegative link weights, the simplest distribution of the link weight $w$ with a distinct different behavior for small values is the polynomial distribution,

$$
F_{w}(x)=x^{\alpha} 1_{x \in[0,1)}+1_{x \in[1, \infty)}, \quad \alpha>0,
$$

where the indicator function $1_{x}$ is one if $x$ is true else it is zero. The corresponding density is $f_{w}(x)=\alpha x^{\alpha-1}, 0<x<1$. The exponent

$$
\alpha=\lim _{x \downarrow 0} \frac{\log F_{w}(x)}{\log x}
$$

is called the extreme value index of the probability distribution. The link weight structure can be controlled by the extreme value index $\alpha$.

(b) Each link is specified by a 2-dimensional link weight vector $\vec{w}(u \rightarrow v)=\left[w_{1}(u \rightarrow v), w_{2}(u \rightarrow v)\right]$, where the component $w_{i}$ is a QoS measure such as delay, jitter, cost, etc. We use SAMCRA [13], a Self-Adapting Multiple Constraints Routing Algorithm to find a shortest or optimal path that satisfies the constraint ${ }^{1}\left[L_{1}, L_{2}\right]$ such that $w_{i}(\mathcal{P})=$ $\sum_{(u \rightarrow v) \in \mathcal{P}} w_{i}(u \rightarrow v) \leq L_{i}$ and minimizes the path length function $l(\mathcal{P})=\max _{1 \leq i \leq 2}\left[\frac{w_{i}(\mathcal{P})}{L_{i}}\right]$. Apart from being attractive in a theoretical analysis, the uniform distribution on $[0,1]$ is the underlying distribution to generate an arbitrary other distribution and is especially interesting for computer simulations [15, Chap. 4]. Furthermore, specific dependencies or correlations exist between QoS measures due to e.g. Weighted Fair Queueing scheduling. Hence, we investigate the link weight structure where the two vector components are correlated uniformly distributed random variables $\in[0,1]$ with correlation coefficient $\rho$ [7]. We tune the correlation coefficient $\rho$ to change the structure of the overlay $G_{\cup s p t}$.

All simulations in the following Sections consisted of generating $10^{4}$ different underlying topologies with link weight structure (a) or (b). In each graph, the overlay $G_{\cup s p t}$ is built up as the union of shortest paths or optimal paths calculated by Dijkstra's algorithm or SAMCRA. Statistic properties of the overlay $G_{\cup s p t}$ such as the degree distribution or the spectrum, the eigenvalues of the adjacency matrix are derived.

\section{UNIFORMLY DISTRIBUTED LINK WEIGHTS}

When $\alpha=1$, the polynomial distribution (1) becomes the uniform distribution, which is equivalent to the link weight tuning scheme (b) with $\rho=1$. In this section, we analyze the structure of the overlay $G_{\cup s p t}$ when the link weights in underlying topology are i.i.d. uniformly distributed.

Theorem 1: For large $N$, the degree distribution in the overlay $G_{\cup s p t}$ on top of the Erdös-Rényi random graph $G_{p}(N)$ with link density $p$ above the disconnectivity threshold $p_{c}$ and equipped with i.i.d. uniformly distributed link weights is

$$
\operatorname{Pr}\left[D_{G \cup \text { spt }}=k\right]=\frac{(-1)^{N-1-k} S_{N-1}^{(k)}}{(N-1) !}
$$

where $S_{N}^{(k)}$ is the Stirling number of the first kind [11].

Proof: See [9]

Conjecture 2: For large $N$, the overlay $G_{\cup s p t}$ on top of the Erdös-Rényi random graph $G_{p}(N)$ with link density $p$ above the disconnectivity threshold $p_{c}$ and equipped with i.i.d. uniform link weights is a connected Erdös-Rényi random graph $G_{p_{c}}(N)$.

Proof: Partially proved in [9]

The simulation result of the degree distribution of $G_{\cup s p t}$ in $K_{N}$ with i.i.d. uniform link weights is shown in Figure 2 (a) and it is nicely matched with the result calculated by Theorem 1. For large $N$ and $p>\frac{\log N}{N}$, in [15, Section 16.3.1], it is shown that also (2) tends to a Poisson distribution

$$
\operatorname{Pr}\left[D_{G \cup s p t}=k\right]=\frac{(-1)^{N-1-k} S_{N-1}^{(k)}}{(N-1) !} \sim \frac{(\log N)^{k}}{N k !}
$$

\footnotetext{
${ }^{1}$ Actually, we choose loose constraints such that an optimal path can always be found via SAMCRA.
} 


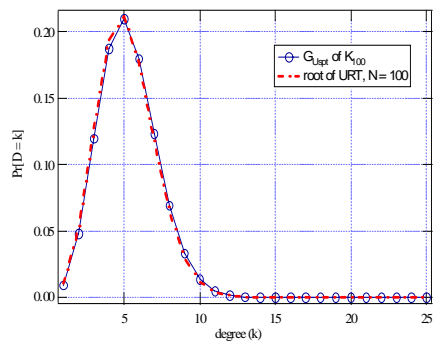

(a)

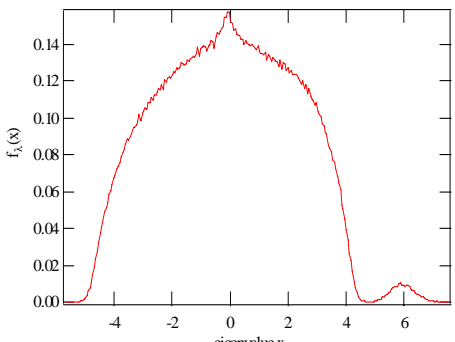

(b)
Fig. 2. Degree distribution and spectrum of the overlay $G_{\cup s p t}$ on top of $K_{100}$ with i.i.d. uniformly distributed link weights.

The binomial degree distribution of the Erdös-Rényi random graph $G_{p}(N)$ also tends to a same Poisson distribution with mean $\log N$. Hence, for large $N$,

$$
\begin{aligned}
\operatorname{Pr}\left[D_{G_{\frac{\log N}{N}}}=k\right] & =\left.\left(\begin{array}{c}
N-1 \\
k
\end{array}\right) p^{k}(1-p)^{N-1-k}\right|_{p=\frac{\log N}{N}} \\
& \sim \frac{(\log N)^{k}}{N k !}
\end{aligned}
$$

The spectrum, the eigenvalues of the adjacency matrix, of $G_{\cup s p t(\alpha)}$ in $K_{100}$ illustrated in Figure 2 (b), is close to the spectrum of a random graph according to the Wigner's Semicircle Law [15, Appendix B]. This correspondence is an additional support for Conjecture 2 .

If Conjecture 2 is correct, the role of the simple ErdösRényi random graph $G_{p}(N)$ may seem to be more important in overlay networks, such as e.g. peer-to-peer networks than in substrate topologies, where only a few complex networks belong to the class of Erdös-Rényi random graphs. Finally, the asymptotic results in this section motivate why a confinement to the complete graph (in later sections) is much less restrictive than it appears at first glance.

\section{ONE DIMENSIONAL LINK WEIGHT TUNING}

In this section, we investigate how the structure of the overlay $G_{\cup s p t(\alpha)}$ varies as we change the extreme value index $\alpha$ of the polynomial distributed link weights. If $\alpha \rightarrow \infty$, it follows from (1) that $w=1$ almost surely for all links. Hence, the overlay $G_{\cup s p t}$ is the same as the underlying topology, since the link weight structure does not differentiate between links. Hence, the $\alpha \rightarrow \infty$ regime is not further considered. When $\alpha=1$, the polynomial distribution (1) becomes the uniform distribution, which has been studied in Section III. In the $\alpha \rightarrow$ 0 regime, all flows are transported over the minimum possible fraction of links in the network: the minimum spanning tree $(\mathrm{MST})^{2}$ [17]. Any failure in a node or link disconnects the MST into two parts and may result in obstruction of transport in the network. The $\alpha \rightarrow 0$ regime may constitute a weak regime although it is highly efficient: only $N-1$ links are used which means that a minimum number of links need to

\footnotetext{
${ }^{2}$ It has been proved that a MST belongs to $G \cup \operatorname{spt}[9]$ and $G \cup s p t$ is always connected. Hence, the event $\{G \cup s p t$ contains $N-1$ links $\}$ is equal to the event $\{G \cup s p t=M S T\}$.
}

be controlled and/or secured. From a traffic engineering point of view, choosing larger $\alpha$ will lead to the use of more paths and, hence, a more balanced overall network load.

Van Mieghem and Magdalena [14] have found that, by tuning the extreme value index $\alpha$, a phase transition occurs around a critical extreme value index $\alpha_{c}$. The critical extreme value index $\alpha_{c}$ is defined as $F_{T}\left(\alpha_{c}\right)=\frac{1}{2}$ where $F_{T}(\alpha)=$ $\operatorname{Pr}\left[G_{\cup s p t(\alpha)}=M S T\right]$. When $\alpha>\alpha_{c}$, the overlay $G_{\cup s p t(\alpha)}$ contains more than $N-1$ links whereas for $\alpha<\alpha_{c}$, all transport traverses a critical backbone consisting of $N-1$ links, which is the $M S T$. Here, we include, besides the complete graphs $K_{N}$ and 2-lattices, but also 3-lattices and Havel-Hakimi [8] power law graphs as underlying topologies.As shown in

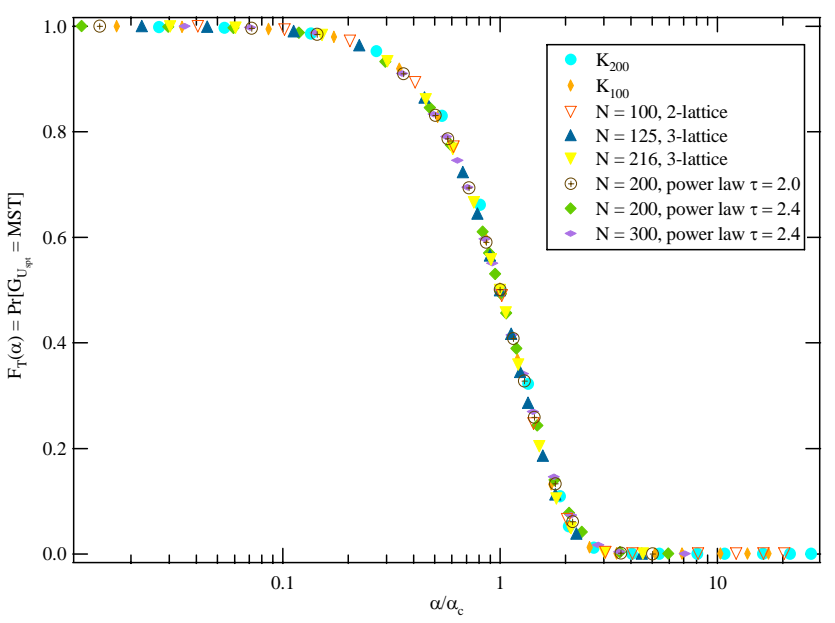

Fig. 3. The probability distribution $F_{T}(\alpha)$ as a function of the normalized $\alpha / \alpha_{c}$.

Figure 3, normalized by $\alpha_{c}$, the same phase transition curve is observed for all these underlying topologies. As $\alpha$ increase, the transport is more likely to traverse over more links and the overlay $G_{\cup \operatorname{spt}(\alpha)}$ is less probably to become a tree. These additional simulations over those reported in [14] strengthen the belief that the curve $F_{T}(\alpha) \approx 2^{-\left(\frac{\alpha}{\alpha_{c}}\right)^{2}}$ is universal for all graphs that are not trees.

Figure 4 demonstrates how the degree in $G_{\cup \operatorname{spt}(\alpha)}$ changes as we tune the extreme value index $\alpha$ of the polynomial distributed link weights. When $\alpha$ is small $\alpha \leq 0.2$, the overlay follows almost the same degree distribution, which decreases even faster than an exponential distribution, the degree distribution of $U R T$ [15]. For a tree structure, the average degree is a constant, $\frac{2(N-1)}{N}$. If the variance of its degree distribution is small, the tree tends to span over longer distance, which can be characterized by the average hopcount of a shortest path. For example, a star has the minimum average hopcount 2 while its degree is either $N-1$ or 1 . Hence, when the overlay $G_{\cup \operatorname{spt}(\alpha)}$ is as sparse as a tree, it is expected to possess less higher degree nodes compared to a $U R T$ and it spans a longer distance. As $\alpha$ increases, more links appear in the overlay, and the nodes tend to have a higher degree. The number of links in the overlay $G_{\cup s p t}$ varies between $N-1$ when $\alpha \rightarrow 0$ and $\frac{N(N-1)}{2}$ when $\alpha \rightarrow \infty$, the overlay $G_{\cup s p t}$ 


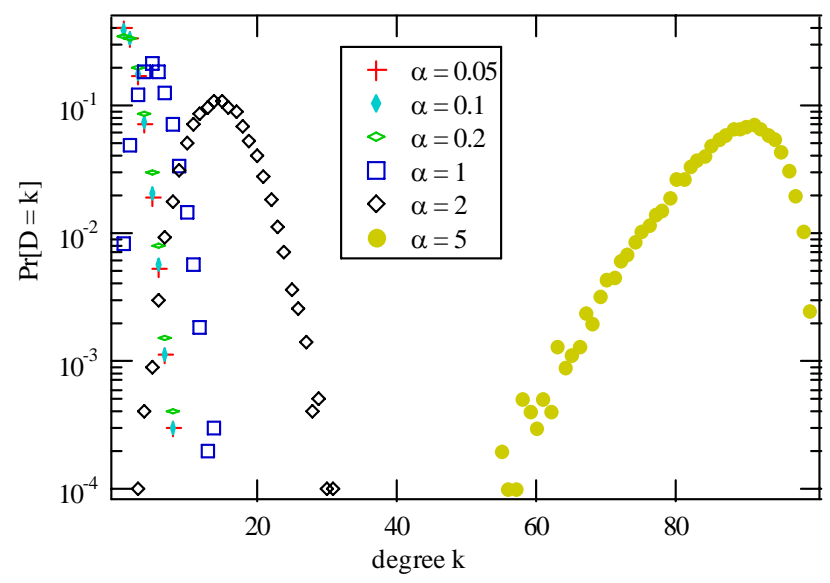

Fig. 4. Degree distribution of the overlay $G \cup s p t$ on top of $K_{100}$ with i.i.d. polynomial link weights.

is equal to the underlying topology.

Simulation results with substrate $K_{N}$ are shown in this section. In fact, when the underlying topology is the ErdösRényi random graph $G_{p}(N)$ with $p<1$, the overlay $G_{\cup s p t}$ structure changes similarly. The overlay $G_{\cup s p t}$ on top of the Erdös-Rényi random graph $G_{p}(N)$ follows the same phase transition as in Figure 3. For large $\alpha$, the link density of the overlay $G_{\cup s p t}$ is limited by the link density of the underlying Erdös-Rényi random graph.

\section{TWO DIMENSIONAL LINK WEIGHT TUNING}

We assign two uniformly distributed link weights with correlation $\rho$ to each link in the underlying graph and the correlation coefficient $\rho$ can be varied within $[-1,1]$. When $\rho=1$, the two link weights of each link are the same, which reduces to the one dimensional uniformly distributed link weight analyzed in Section III. When $\rho=-1, \vec{w}(u \rightarrow v)=$ $\left[w_{1}(u \rightarrow v), w_{2}(u \rightarrow v)\right]=\left[w_{1}(u \rightarrow v), 1-w_{1}(u \rightarrow v)\right]$. We recall the definition [13] of the path length function $l(\mathcal{P})=$ $\max _{1 \leq i \leq 2}\left[\frac{w_{i}(\mathcal{P})}{L_{i}}\right]$. Since we assume the same constraint for these two link weight measures $L_{1}=L_{2}$, which are large such that the shortest path always satisfies the constraints. Hence, the path length function to find the optimal path can be reduced to $l(\mathcal{P})=\max \left(\begin{array}{l}w_{1}(\mathcal{P}) \\ w_{2}(\mathcal{P})\end{array}\right)$, where $w_{i}(\mathcal{P})=\sum_{(u \rightarrow v) \in \mathcal{P}} w_{i}(u \rightarrow v)$. The path length of a $h \geq 2$ hop path is

$$
l_{h}(\mathcal{P})=\max \left(\begin{array}{l}
w_{1}(\mathcal{P}) \\
w_{2}(\mathcal{P})
\end{array}\right)=\max \left(\begin{array}{c}
w_{1}(\mathcal{P}) \\
h-w_{1}(\mathcal{P})
\end{array}\right) \geq \frac{h}{2} \geq 1
$$

while the path length of a one hop path

$$
l_{1}(\mathcal{P}=u \rightarrow v)=\max \left(\begin{array}{c}
w_{1}(u \rightarrow v) \\
1-w_{1}(u \rightarrow v)
\end{array}\right)<1 \leq l_{h}(\mathcal{P})
$$

Hence, when $\rho=-1$, the link between the source and destination, if exists, is always the shortest path. All links in the underlying graph will appear in the overlay $G_{\cup s p t}$. In other words, the overlay $G_{\cup s p t(\rho=-1)}$ is the same as the substrate, which corresponds to $G_{\cup \operatorname{spt}(\alpha \rightarrow \infty)}$ in Section IV.

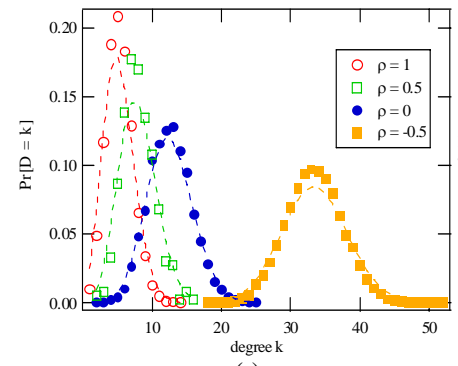

(a)

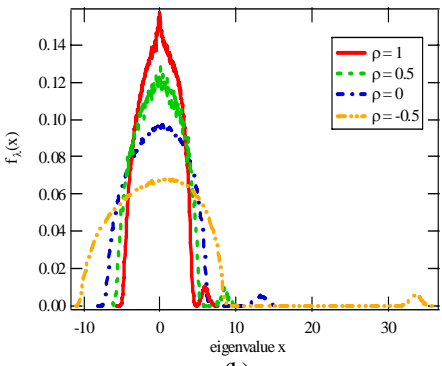

(b)
Fig. 5. Degree distribution and spectrum of the overlay $G_{\cup s p t}$ in $K_{100}$ with 2-dimensional correlated uniformly distributed link weights.

When the underlying topology is the complete graph, the degree distribution of the overlay $G_{\cup s p t}$ displayed in Figure 5(a) is close to the binomial distribution, the degree distribution of an Erdös-Rényi random graph. The dotted line is the degree distribution of the Erdös-Rényi random graph $\operatorname{Pr}[D=k]=\left(\begin{array}{c}N-1 \\ k\end{array}\right) p^{k}(1-p)^{N-1-k}$ where $p$ is set as the link density ${ }^{3}$ of the corresponding overlay $G_{\cup \operatorname{spt}(\rho)}$. As shown in Figure 5(b), the spectrum of the overlay $G_{\cup \operatorname{spt}(\rho)}$ with different correlation coefficient $\rho$ is close to the spectrum of an Erdös-Rényi random graph according to the Wigner's Semicircle Law [15, Appendix B]. Both the degree distribution and the spectrum indicate that the overlay $G_{\cup s p t(\rho)}$ on top of $K_{100}$ with 2-dimension correlated uniform link weights is close to an Erdös-Rényi random graph. The same behavior has been observed when the substrate is not the complete graph but the Erdös-Rényi random graph: the overlay $G_{\cup s p t(\rho)}$ is also always close to an Erdös-Rényi random graph.

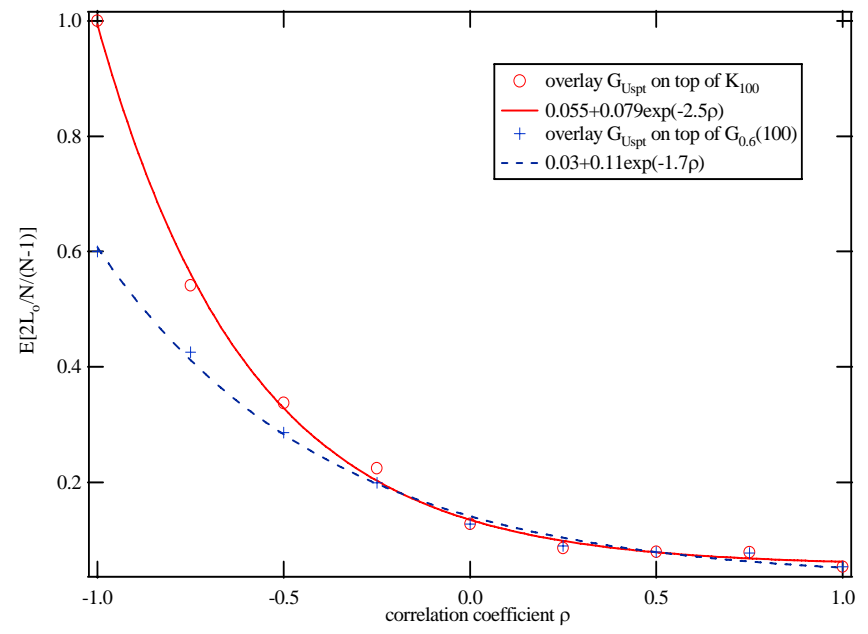

Fig. 6. Link density of the overlay $G \cup s p t$ on $K_{100}$ and $G_{0.6}(100)$ with 2-dimensional correlated uniformly distributed link weights.

The link density of the overlay $G_{\cup s p t}$, the number of links $L_{o}$ in the overlay $G_{\cup s p t}$ divided by the maximum number of links in a graph $\frac{N(N-1)}{2}$, is plotted in Figure 6. When

\footnotetext{
${ }^{3}$ It is the average link density $E\left[\frac{2 L_{o}}{N(N-1)}\right]$ of the $10^{4}$ generated overlay $G \cup s p t$ in each simulation, where $L_{o}$ is the number of links in the overlay $G \cup s p t$.
} 
$\rho=-1$, the overlay $G_{\cup s p t(\rho=-1)}$ is equal to the substrate $G_{p_{s}}(N)$, where $p_{s}$ is the link density of the underlying ErdösRényi random graph. The link density of the overlay is then determined by the link density of the underlying topology. According to conjecture 2, when $\rho=1$, the overlay $G_{\cup s p t}$ is a connected Erdös-Rényi random graph $G_{p_{c}}(N)$ with link density $p_{c}$, which is independent of the link density of the substrate. The link density of the overlay $G_{\cup s p t(\rho)}$ decreases exponentially from $p_{s}$ the link density of the substrate to $p_{c} \sim$ $\frac{\log N}{N}$ as a function of the correlation coefficient $\rho$.

\section{CONCLUSION}

Taking into account both current best-effort routing and QoS routing, we construct two kinds of overlay networks: $G_{\cup s p t(\alpha)}$ by tuning the extreme value index $\alpha$ of polynomial link weights and $G_{\cup s p t(\rho)}$ by tuning the correlation coefficient $\rho$ of the 2-dimensional correlated uniformly distributed random link weights. The observation of the structural transitions of the overlay $G_{\cup s p t}$ as shown in the table below is considered as the main contribution of this paper.

\begin{tabular}{|l|l|}
\hline one dimensional $\alpha \in[0, \infty)$ & two dimensional $\rho \in[-1,1]$ \\
\hline$G_{\cup s p t\left(\alpha<\alpha_{c}\right)}=M S T$ & \\
\hline$G_{\cup s p t(\alpha=1)} \simeq G_{p_{c}}(N)$ & $G_{\cup s p t(\rho=1)} \simeq G_{p_{c}}(N)$ \\
\hline$G_{\cup s p t(1<\alpha<\infty)} \neq G_{p}(N)$ & $G_{\cup s p t(-1<\rho<1)} \simeq G_{p}(N)$ \\
\hline$G_{\cup s p t(\alpha \rightarrow \infty)}=G_{p_{s}}(N)$ & $G_{\cup s p t(\rho=-1)}=G_{p_{s}}(N)$ \\
\hline
\end{tabular}

The overlay $G_{\cup s p t}$ as defined is always connected. With one dimensional link weights tuning, the overlay $G_{\cup \operatorname{spt}(\alpha)}$ varies from the sparsest structure (the $M S T$ ) to the densest, the substrate $G_{p_{s}}(N)$. In a two dimensional link weight tuning, the overlay $G_{\cup s p t(\rho)}$ is always close to the Erdös-Rényi random graph. The role of the simple Erdös-Rényi random graph $G_{p}(N)$ may seem to be more important in overlay networks formed on dense substrates such as popular peerto-peer networks. The link density of $G_{\cup s p t(\rho)}$ varies within $\left[\frac{\log N}{N}, p_{s}\right]$, which is smaller than the link density range of $G_{\cup \operatorname{spt}(\alpha)}:\left[\frac{2}{N}, p_{s}\right]$.

The understanding of the overlay structure with variable link weight structure points to the possibility to control the network structure or to steer or balance transport by tuning the link weight structure. Here, we focus on homogeneous link weight structures, and more studies on heterogeneous link weight tuning can be interesting. Apart from the theoretical investigation provided in this paper, further examination about feasibility and scalability of link weight tuning in real large networks is needed.

\section{ACKNOWLEDGEMENT}

We greatly appreciate the comments from F.A. Kuipers on the overlay construction with QoS routing. This research was supported by the Netherlands Organization for Scientific Research (NWO) under project number 643.000.503 and the European Union CONTENT NoE (FP6-IST-038423).

\section{REFERENCES}

[1] A. Clauset and C. Moore, Accuracy and Scaling Phenomena in Internet Mapping, Phys. Rev. Lett. 94, 018701 (2005).

[2] A. Ganesh, L. Massoulie and D. Towsley, "The effect of network topology on the spread of epidemics", Proc. IEEE Infocom, 2005.

[3] A. Lakhina, J. Byers, M. Crovella and P. Xie, "Sampling Biases in IP Topology Measurements", Proc. of IEEE INFOCOM, San Francisco, CA, 2003.

[4] B. Bollobas, Random Graphs, 2nd ed., Cambridge University Press, Cambridge, 2001.

[5] D. Achlioptas, A. Clauset, D. Kempe and C. Moore, On the bias of traceroute sampling: or, power-law degree distributions in regular graphs, Proc. of the thirty-seventh annual ACM symposium on Theory of computing, Baltimore, MD, USA, 2005.

[6] E. W. Dijkstra, "A note on two problems in connection with graphs", Num. Math., 1:269-271., 1959.

[7] F.A. Kuipers and P. Van Mieghem, "Conditions that Impact the Complexity of QoS Routing", IEEE/ACM Transaction on Networking, vol. 13, no. 4, pp. 717-730, August 2005.

[8] G. Chartrand and L. Lesniak, Graphs and Digraphs, Chapman and Hall/CRC, 1996.

[9] H. Wang and P. Van Mieghem, "Properties of the Observable Part of a Network", unpublished.

[10] L. Zhao, K. Park, and Y.-C. Lai, "Attack vulnerability of scale-free networks due to cascading breakdown", Phys. Rev. E 70, 035101(R), 2004.

[11] M. Abramowitz and J. A. Stegun, Handbook of Mathematical Functions, Dover Publications Inc., New York, 1999.

[12] M. Castro, M. Costa, and A. Rowstron. Should we build Gnutella on a structured overlay. ACM SIGCOMM Computer Communications Review 34(1), pp. 131-136, 2004.

[13] P. Van Mieghem and F.A. Kuipers, "Concepts of Exact Quality of Service Algorithms", IEEE/ACM Transaction on Networking, vol. 12, no. 5, pp. 851-864, October 2004.

[14] P. Van Mieghem, and S. M. Magdalena, "A Phase Transition in the Link Weight Structure of Networks", Phys. Rev. E 72, 056138, 2005.

[15] P. Van Mieghem, Performance Analysis of Communications Systems and Networks, Cambridge University Press, 2006.

[16] P. Van Mieghem, Data Communications Networking, Techne Press, 2006.

[17] P. Van Mieghem and S. van Langen, "Influence of the link weight structure on the shortest path", Physical Review E 71, 056113, 2005.

[18] R. Hekmat and P. Van Mieghem. Connectivity in wireless ad hoc networks with a log-normal radio model. Mobile Networks and Applications, The Journal of Special Issues on Mobility of Systems, Users, Data and Computing, 2005.

[19] R. T. Smythe and John C. Wierman. First-Passage Percolation on the Square Lattice. Springer, Berlin Heidelberg New York, 1978.ev. E 66, 046106, 2002.

[20] R. van der Hofstad, G. Hooghiemstra, and P. Van Mieghem, "First passage percolation on the random graph", Probability in the Engineering and Informational Sciences (PEIS), Vol. 15, pp. 225-237, 2001.

[21] S. Androutsellis-Theotokis and D. Spinellis, "A survey of peer-to-peer content distribution technologies", ACM Computing Surveys (CSUR), 36(4):335 - 371, December 2004.

[22] T. Watanabe, Y. Higashi, and A. Nakamura, "An approach to robust network construction from graph augmentation problems", In Proc. of 1990 IEEE Int'l Symp. on Circuits and Systems, pages 2861-2864, 1990. 